\title{
ABSTRACTS
}




\title{
Epidemiology
}

Acta Genet Med Gemellol 43: 107 (1994)

(C) 1994 by The Mendel Institute

International Congress

40th Anniversary of The Mendel Institute

\section{Studio Epidemiologico del Nato da Gravidanza Plurima}

\author{
D. Cianciulli, M. Pezzati, C. Carbone, G. Mainardi, C. Panero, E. Biagioli- \\ Cosenza, R. Biadaioli, A. La Torre \\ Dipartimento di Pediatria - Unità Operativa di Neonatologia, Università degli Studi di Firenze, \\ Italia
}

\begin{abstract}
La gravidanza gemellare è stata sempre considerata «a rischio» come dimostrato dalla elevata incidenza di mortalità e morbosità. L'entità del rischio è stata valutata con una indagine statistica condotta sui nati nel periodo 1987-1993 nella U.O. di Neonatologia annessa alla Clinica Ostetrico-Ginecologica dell'Università di Firenze. Vengono riferiti i risultati di uno studio relativo all'incidenza di patologia in 463 gemelli confrontata con quella osservata in 15,658 nati da parto singolo. L'incidenza della gemellarità è stata del $2.87 \%$ (la media nazionale si aggira intorno al $2 \%$ ). L'età gestazionale media dei gemelli oscilla intorno alle 35 settimane. Il $38 \%$ dei gemelli presenta un peso alla nascita $\geq 2,500 \mathrm{~g}$. rispetto al $94 \%$ dei singoli; il $12 \%$ dei gemelli è nato con peso $<1,500 \mathrm{~g}$. rispetto all' $1.5 \%$ dei singoli. Il basso peso dei gemelli alla nascita è il risultato della prematurità e del ritardo di accrescimento. L'evento malformativo nella nostra U.O. è stato del $2.98 \%$ nelle gravidanze singole e del $5.61 \%$ nelle gravidanze plurime. La mortalità neonatale precoce è stata del $4.89 \%$ nei gemelli contro lo $0.46 \%$ nei singoli. La mortalità perinatale è stata del $7.77 \%$ nei gemelli contro 1 ' $1.15 \%$ nei singoli. La patologia respiratoria ha inciso nel parto singolo per il $2.31 \%$ e nel parto plurimo per il $4.96 \%$. I risultati della nostra indagine sono stati discussi e confrontati con quelli della letteratura.
\end{abstract}

\section{(Presentato come poster)}

Per Corrispondenza: Dr. D. Cianciulli, U.O. Neonatologia - Maternità, Via Morgagni 85, 50100 Firenze, Italia. 